Indexaciones: Repositorio de Revistas UCR, DIALNET, Latindex, REDALYC Directorio y recolector de recursos digitales del Ministerio de Cultura de España, Directory of Open Access Journals. Diálogos Revista Electrónica de Historia ISSN 1409- 469X. Número especial 2008. Dirección web: http://historia.fcs.ucr.ac.cr/dialogos.htm

\section{Teatro de vecindario: grupos de teatro comunal del valle central de Costa Rica, 1975-1990.}

\section{Mario Salazar Montes}

Institución: Estudiante de la escuela de Historia de la Universidad de Costa Rica Teléfonos: 88155082 / 222140663

Correos electrónicos: ucrtallerhistoria@gmail.com montesalmar@gmail.com 


\section{Introducción}

A Todos los integrantes de los grupos de teatro vecinal. A las personas que participaron en la lucha contra el TLC-CR-EU.

A mi abuelo Paco, entre infinitas cosas, por haberme enseñado la dimensión universal de lo cotidiano

En el mes de marzo de 1990, Alejandro Tosatti, publicó un artículo en la revista Aportes donde cuenta la experiencia artística que ha desarrolló un grupo comunal de Alajuela:

La niña de los ojos grandes se ha puesto de pie y mira emocionada las evoluciones de una bailarina. “Cuando sea grande quiero ser como ella”, dice. Estamos en el INVU Las Cañas de Alajuela; [...] La reacción de la niña es solo un ejemplo del creciente gusto y aceptación de las diferentes disciplinas artísticas, en una comunidad que no se diferencia en nada de muchas otras comunidades y barriadas que se han ido expandiendo como manifestación física del proceso de urbanización. En nada excepto que aquí, en el INVU Las Cañas, surgió desde hace años un grupo que trabaja intensamente para la superación cultural y artística de sí mismo y de la comunidad $^{1}$

La experiencia que relata Tosatti, contrario de lo que señala, no fue para nada excepcional en muchas comunidades de Costa Rica durante las décadas de 1970 y 1980, sin embargo, la actividad descrita se da en un momento en que el auge de este tipo de manifestaciones artístico-comunales empezaba a desaparecer. Antes de analizar cómo fue que una actividad comunal tan importante como la que realizó el grupo Antorcha de Alajuela en 1990 no tuvo continuación, como sucedió en muchas otras comunidades con experiencias similares, queremos preguntarnos:

1. ¿Cómo fue que este tipo de agrupaciones surgieron?

2. ¿Quienes fueron los actores, actrices y artistas comunales que participaron en los grupos teatrales comunitarios?

3. ¿Que metodologías, obras de teatro y dinámicas particulares se desarrollaron al interno de los grupos?

4. ¿Cuáles fueron los espacios de representación y que tipo de público que asistía a sus presentaciones?

A partir de dichas interrogantes, intentaremos plantear un acercamiento al fenómeno del teatro vecinal en el valle central de Costa Rica durante las décadas de 1970 y 1980.

\footnotetext{
1 "La fiesta anual del grupo Antorcha. Entretelones de una red cultural”, Aportes, Marzo de 1990, p 23.
} 


\section{Surgimiento de los teatros comunales en Costa Rica en 1970-1980.}

En 1972, un delegado importante de la UNESCO visitó Costa Rica, con el propósito de intención de activar y promover una politica cultural que se propusiera el acercamiento de públicos nuevos, se trataba de Fabio Pacchioni. Su propuesta era concretamente una transformación de la actividad teatral en Costa Rica. En términos generales, el ambicioso proyecto de la UNESCO consistía en modificar el clásico concepto de cultura con que habían desarrollado su política cultural las elites gobernantes de los países latinoamericanos. Estos delegados culturales fueron conocidos como los "expertos de la UNESCO”. ${ }^{2}$

Por motivo de las dictaduras latinoamericanas, muchos artistas, buscaron asilo en nuestro territorio y más temprano que tarde, se van acercando e incorporando al medio artístico de Costa Rica. Desde un primer momento estos artistas pusieron a los nacionales en contacto con la nueva dramaturgia latinoamericana. ${ }^{3}$

Bastante tiempo antes de que estos fenómenos se desarrollaran, en las comunidades de Costa Rica se estimuló la creación de actividades escolares y religiosas donde los alumnos o feligreses invitaban a sus padres y a la comunidad a una serie de actividades que tenía como propósito la recaudación de fondos por medio de venta de comidas y de actividades culturales. Estas denominadas "veladas escolares" son un fenómeno de particular importancia para el desarrollo del teatro comunal. Por medio de esta experiencia que atraviesa buena parte del siglo XX, se desarrolló un formato de representación escénica que teatraliza las "estampas típicas" de la literatura costumbrista. Dos son las razones por las que nos parece un elemento determinante para el surgimiento de un teatro vecinal: por un lado, crea una larga experiencia artístico comunal y por otro lado, crea una estética vecinal, con la que la comunidad se identifica. También nos parece que la popularidad que tienen en las comunidades las "Veladas Escolares", antes de la década de 1970, problematiza la idea de que es con la aparición del Ministerio de Cultura, Juventud y Deportes (MCJD) y las políticas que este desarrolla, que se inicia y populariza la representación en Costa Rica.

Durante la segunda mitad del siglo XX, Costa Rica desarrolló una fuerte organización a nivel comunal que luchó porque una serie de derechos ciudadanos fueran cumplidos, y veló por el buen 2 Cuevas Rafael, El punto sobre la i. Políticas culturales en Costa Rica (1948-1990), (San José, MCJD, 1996), p159

3

Fumero Patricia, “Teatro contemporáneo costarricense: 1970-2000”, p 7 
funcionamiento de los servicios públicos, pero sobre todo por que estos servicios no cometieran ningún tipo de abusos contra la comunidad, como subidas de precios. En el desarrollo de esta experiencia de organización comunal costarricense fue de suma importancia el liderazgo que ejercieron las bases del Partido Comunista y de otras agrupaciones de izquierda, que en el caso del primero, en un primer momento al estar proscrito, ve en este espacio, un lugar fundamental para desarrollar nuevas luchas. ${ }^{4}$

En los grupos de teatro comunal analizados para esta época apreciamos una participación importante de cuadros de la izquierda. Esto no quiere decir que los grupos estaban constituidos solo por integrantes de la izquierda, sin embargo éstos le daban un carácter determinado a los grupos donde participaban. Al mismo tiempo, algunas organizaciones religiosas también fomentaron e incentivaron la participación y la integración de grupos de teatro y de actividades culturales en las comunidades.

En la visita que el experto de la UNESCO realizó al país 1972 promueve una propuesta que denomina "Viaje de ida y vuelta al público", en ella se plantea el desarrollo y la práctica de una política que transformara al teatro en un movimiento profesional y aficionado, que desarrollara un público más amplio. Sin embargo, es Oscar Castillo, director de la Compañía Nacional Teatro (CNT) durante ese periodo, quien acoge y materializa la propuesta del especialista de la UNESCO.

De esta manera queda instaurada, a través del Estado costarricense, una estructura que empezó a promover grupos de teatro aficionado en tres áreas específicas: las comunidades, las empresas, ya sean agrícolas o industriales, y las instituciones del Estado. En un informe anual de la CNT, publicado en la revista Escena a finales de 1980, se señala lo siguiente:

Fue así como nació en el año de 1977 el Taller Nacional Teatro con el objetivo, por un lado, de formar actores profesionales, $y$, colateralmente, capacitar a personal especializado en la promoción teatral. ${ }^{5}$

Entre 1970 y 1975 se crearon 16 grupos de teatro comunal, 18 grupos de teatro en colegios. ${ }^{6} \mathrm{Y}$ en el informe de anual de 1980 de la CNT se infiere del documento, la promoción en 27 lugares

$4 \quad$ Alvarenga, Patricia. De vecinos a ciudadanos. Ed. EUNA y EUCR. 2005

5 Compañía Nacional de Teatro. Programa de Trabajo Repertorio 1980. Escena n3, p3

$6 \quad$ Patricia Fumero, “Teatro contemporáneo costarricense: 1970-2000”, p7 
distintos, aunque no se especifica el grupo. ${ }^{7}$ Hay que hacer la salvedad de que estos 27 lugares que tienen un promotor, varios son alumnos del TNT que están haciendo práctica pedagógica. Véase cuadro 1.

Una preocupación subyacente en esa coyuntura fue desarrollar un "Teatro Popular”. Con motivo de la celebración de los 10 años de la fundación de la CNT, Aportes le realizó una entrevista a Adriana Prado, Directora de la institución quien entre otras cosas señala lo siguiente:

Por eso es importante uno de nuestros principales programas que lo constituye el Taller Nacional de Teatro que ya graduó sus primeros alumnos. Con esto, promoveremos ese teatro popular,...que los grupos se organicen en el campo, las fábricas los colegios y por medio del teatro expresen su realidad, la puedan objetivar al verla representada y escriban también sus obras. ${ }^{8}$

Como señala Adriana "Mimí” Prado, la intención de que los teatros comunitarios sirvan como un instrumento para representarse, denunciar y señalar las realidades propias de los obreros, de las comunidades y de los estudiantes, se encuentra desde las posiciones directivas de la CNT. Este hecho señala ya una nueva reconceptuaización de los conceptos de arte y cultura que tradicionalmente se habían aplicado en Costa Rica. Como lo ha señalado George Yúdice, en este escenario la "Cultura" es un recurso al que se apela desde la política o desde la economía para dirimir conflictos. ${ }^{9}$

Por otra parte queremos reseñar la experiencia de un grupo de teatro comunal que tiene un desarrollo aparte: el grupo experimental Éxodo. Éxodo fue un grupo de teatro comunal de los barrios del sur de la capital. Estaba compuesto por alumnos de la primera generación del TNT y contó con cuatro integrantes: Lorena Delgado, Rubén Garro, Víctor Valdelomar y Magda Vargas. Lo singular del surgimiento de este grupo es que nace a partir de un grupo de teatro comunal que se desarrolla en Paso Ancho con el nombre de "Barrio Pobre", al interno del Movimiento Juventud Unida de Paso Ancho (MOJUPA). Este grupo nace a partir de la experiencia de los vecinos que participaron previamente en las "Veladas Escolares" que se realizaban en la comunidad. En la década de 1970, esta experiencia estético-vecinal, va a adquirir un alto grado de politización, en parte por el trabajo realizado por MOJUPA en esta comunidad. Como señala Víctor Valdelomar

7

8 Los 10 años de la Compañía. En busca de la identidad del Teatro Costarricense. Aportes, Abril-mayo de 1981, p20

9 Yúdice George. La cultura como recurso. Barcelona España. Ed. Gedisa. 2002 
en la entrevista realizada, este grupo surge de

...la casa de la juventud que funda el Movimiento Nacional de Juventudes en Paso Ancho. Varios dirigentes comunales empezaron eso, jóvenes, empezaron la formación de la casa de la juventud y en la casa de la Juventud se formo el grupo de teatro, que inicialmente hacían, lo que hacen mucho de estos grupos aficionados, que lo que hacen son, lo que llaman: "Estampas Típicas": "el Matrimonio Campesino" y ese tipo de cosas. Pero la época tiene muchas cuestiones sociales que empiezan a influir en los jóvenes sobre todo. Entonces había una inquietud por hablar sobre el problema de la drogadicción sobre el problema en general de las situaciones de las comunidades [...] [El origen del grupo] es más de tradición, en nuestras comunidades, en los barrios del sur, en algunos lugares que yo trabaje, por ejemplo San Juan de Dios de Desamparados, había una tradición teatral de muchísimos años, que era la gente que se encargaba de hacer las "Veladas Escolares". Cuando se organizaban las "Veladas Escolares", entonces ellos hacían los cuadros estos típicos y estas cosas. En Paso Ancho también. Esta tradición del grupo teatral Barrio Pobre venia mucho de gente ya vieja que hacían teatro de este tipo. ${ }^{10}$

Además, el tránsito de "Barrio Pobre" a Éxodo no estuvo exento de conflicto como también nos señalo Valdelomar, ya que algunos integrantes de "Barrio Pobre”, no querían profesionalizar el grupo, reivindicando una experiencia escénica más tradicional e incluso comunitaria:

Hubo algunos roces con los dirigentes que querían seguir, digamos en una, en ese tipo de actividad muy aficionada y no querían pues, que hubiera una preparación en el grupo. ${ }^{11}$

\section{-Los promotores teatrales}

Los promotores teatrales fueron algunos de los artistas de esta época que estuvieron más cerca de las comunidades y de las problemáticas y luchas que éstas llevaban a cabo. Es cierto que también muchos grupos de teatro independiente llevaron el teatro a las comunidades, pero ninguno como los promotores estuvo tan cerca de estas comunidades como ellos. En la mayoría de los casos, los promotores desarrollaban un trabajo de meses en una o varias comunidades al mismo tiempo. Pero ¿Quiénes eran los promotores? ¿En que lugares trabajaron? Son preguntas básicas que tenemos que responder para acercarnos a estos funcionarios de la CNT.

De la primera generación del TNT que inició sus estudios en 1977 se graduaron 17 personas. En el informe anual de 1979 se señala que ese año se contó, con 16 promotores estables, sin embargo, más adelante el mismo documento acota que en "servicio" se haya trabajando once 10 Entrevista realizada a Víctor Valdelomar en la UCR, San Pedro, 9 de octubre del 2007 11 Ibíd. 
promotores $^{12}$. Después de que se gradúan del TNT, muchos de estos artistas son incorporados al Departamento de Promociones de la CNT, cuando ésta era dirigida por Mimí Prado.

En esta primera etapa, la promoción teatral va tener un periodo de cuatro años, que coinciden con los de la administración Carazo Odio, donde el impulso a la promoción teatral y a la creación de grupos de teatro comunal, de obreros, de estudiantes y de empleados públicos es muy fuerte. En 1981, tres años después de que los promotores teatrales iniciaran su labor en los diferentes lugares donde trabajaron, se empiezaron a recibir los primeros frutos. En los días 26, 27 y 28 de junio de ese año, se lleva a cabo una "Muestra de teatro de comunidades y grupos aficionados", organizada por el Departamento de Promociones de la CNT. "La actividad tenía como fin principal dar a conocer la labor de los grupos de teatro aficionado que funcionan en el país". ${ }^{13}$ En la revista Escena, el coordinador del Departamento de Promociones del MCJD, señala que en la muestra se presentaron 13 grupos de teatro comunal que "tenían la característica común de realizar un trabajo permanente y un seguimiento igual por parte de los promotores de la CNT”. ${ }^{14}$ A partir de este artículo que realiza Miguel Díaz, coordinador de promotores, podemos apreciar la variedad de comunidades y de grupos que se presentaron. Véase cuadro 2.

Es importante mencionar que durante estos cuatro años también encontramos promotores que no se dedicaron a la promoción teatral si no más bien a la promoción cultural, lo que implicaba un trabajo distinto porque estos, no se especializaban en promover grupos de teatro comunal, si no que promovían otras actividades artísticas. Sin embargo, este tipo de promotor cultural es el que va a tomar más auge en los años posteriores a este primer ciclo de cuatro años, sin que tampoco dejaran de existir los promotores teatrales propiamente dichos.

De los cinco promotores a los que entrevistamos (no todos son de la primera generación del TNT o del TNT), notamos que cuando empezaron su participación como promotores teatrales o culturales, no sobrepasaban los 23 años de edad, nos atrevemos asegurar que esta ambiciosa empresa fue encargada a generaciones muy jóvenes del medio artístico, que además veían en este proyecto una oportunidad para incorporarse laboralmente al Estado, en un momento marcado por una crisis económica muy fuerte. Esta ardua labor continua e ininterrumpida realizada por los promotores sobrepasaba el cuarto de tiempo que se les pagaba como salario. En algún momento se señaló la necesidad de aumentar este cuarto de tiempo y otras reivindicaciones, pues esto

12 Compañía Nacional de Teatro. Labor del Año 1979. Montajes, Escena n3, p7.

13 Hacia un Teatro Popular Costarricense, Escena n6, p19

14 Hacia un Teatro Popular Costarricense, Escena n6, p20 
influye en la atención a los grupos, y en última instancia a su derecho de hacer teatro. ${ }^{15}$

Varios promotores que entrevistamos provenían de los barrios del sur de San José. Es muy interesante que en el caso de Víctor Valdelomar, Rubén Garro, Lorena Delgado y Magda Vargas, habían empezado su labor artístico-comunal paralelamente a los estudios que ellos realizaban en el TNT, por medio del grupo experimental Éxodo, que como señala Víctor Valdelomar, surge: ...de una inquietud evidentemente política, [...] de digamos, de un desarrollo de conciencia social en las comunidades. Y como todos los miembros somos de los Barrios del Sur de San José, nos agrupamos en el Centro Episcopal de Barrio Cuba y ahí empezamos una actividad con varios montajes [...] estuvimos muy cerca de lo que se llamo, la teología de la liberación ${ }^{16}$ Al respecto Rubén Garro también nos constata "que la idea era que Éxodo fuera y se proyectara a nivel de los barrios del Sur" ${ }^{17}$. Nos parece que la experiencia que estos artistas desarrollaron en este grupo va ser determinante en los proyectos que posteriormente cada uno desarrolla.

En el caso de Fernando Vinocour su experiencia es distinta pues este promotor no estudia en el TNT, si no en la Escuela de Artes Dramáticas de la Universidad de Costa Rica y al finalizar la década de 1970 desarrolla una experiencia de teatro político, popular y callejero, en una primera etapa, a través del “Teatro Carpa”, fundado por Alfredo Catania. El “Teatro Carpa” giraba por comunidades presentando sus espectáculos. Posteriormente Vinocour pasa a formar parte del grupo independiente "La Trama”, fundado por estudiantes de la escuela de artes dramáticas de la UCR. La trama desarrolla un tipo de teatro como “....acontecimiento público. Pues comenzaron a montarse obras en espacios como la Universidad de Costa Rica, las plazas de la ciudad, etc.” ${ }^{18}$ A partir del desarrollo de temáticas políticas. Y finalmente el caso de Marco Araya, que procede de Alajuela y es en esta ciudad donde desarrolla su trabajo como promotor. Araya, nos señaló que sus experiencias previas a los estudios teatrales las adquirió en una agrupación religiosa que tenía un grupo de teatro, lo que viene a demostrar el importante papel jugado por varias instituciones religiosas en el desarrollo o estimulación del teatro. Podemos señalar que la función del promotor esta atravesada por su carácter institucional pero al mismo tiempo, por su experiencia comunitaria, universitaria o religiosa. De esta forma, es en los promotores teatrales donde la institucionalidad y la comunidad se encuentran.

15 Hacia un Teatro Popular Costarricense, Escena n6, p19Ibíd. p20

16 Entrevista realizada a Víctor Valdelomar en la UCR, San Pedro, 9 de octubre del 2007

17 Entrevista realizada a Ruben Garro, en la UCR, 13 de setiembre del 2007

18 Fernando Vinocour editor, La tradición del presente. San José Costa Rica, ed. Perro Azul, 2007, p126 


\section{-El discurso comunal tras las temáticas artíticas.}

Hasta el momento hemos venido analizando el rol del Estado en el desarrollo de una actividad teatral comunal muy dinámica, sin embargo no hemos analizado el papel que jugaron las comunidades para desarrollar una independencia. El asunto fundamental que queremos plantear es ¿cómo entender esta independencia, si de ante mano sabemos que lo promotores teatrales jugaron un papel determinante en estos grupos?

En pocas palabras, el grado de independencia que desarrollan los grupos se logra a través de la libertad temática que estos tienen para plantear y representar, a partir del teatro, las problemáticas de sus comunidades. Podemos asegurar que en ninguna experiencia de las que analizamos estuvo presente algún tipo de censura al trabajo que cada grupo desarrollaba desde la realidad de cada comunidad.

De ante mano sabemos que los promotores trabajan para el Estado, sin embargo un fenómeno muy interesante es que muchos de estos “empleados públicos” con sus características particulares, o provenían de partidos de izquierda o tenían sensibilidades sociales adquiridas a través de instituciones religiosas o a través de una politización que adquieren en las universidades públicas, donde para esta época existía un alto nivel de politización. Por otro lado, como hemos demostrado, muchos de estos promotores eran vecinos de las mismas comunidades donde trabajan. En los casos donde no sucedió esto, los promotores no interfirieron en el planteamiento de estas temáticas.

Por otro lado, en muchas de estas agrupaciones de teatro de comunidad se uso el método de la Creación Colectiva, la cual creemos que es un factor o un instrumento que al utilizarse logra ir permeando el trabajo artístico de los grupos de teatro comunal de una realidad percibida y construida entre los miembros que integran el grupo. Siendo la mayoría de los artistas de una comunidad específica, este método, y un alto nivel de politización, permiten que los grupos manejen una independencia a la hora de simbolizarse como comunidad.

Como veremos más adelante, cuando analicemos las obras de algunos de estos grupos, en la mayoría de los casos están basadas en las problemáticas de cada comunidad. Esto incluso representó un problema cuando los grupos trascendieron del espacio local propio a espacios regionales, nacionales o internacionales, como lo señala Alexander Valverde del grupo Caña Brava. Según nos cuenta, cuando algunas de las obras que salieron del contexto de la comunidad 
de San Juan de Dios Desamparados a otras comunidades, por medio de los festivales que se realizaban, hubo que cambiarles partes, para que se entendiera o para que las obras tuvieran un alcance más regional. ${ }^{19}$

Reiteramos que en el caso de la experiencia de los grupos de teatro comunal hubo una independencia en el desarrollo de un discurso que para nosotros es fundamental para que una agrupación pueda denominarse independiente. En algunos casos esto fue tan claro que muchas obras creadas por estas agrupaciones tentaron, a través de las temáticas planteadas, al mismo poder represor del Estado, como lo veremos más adelante. Al mismo tiempo reconocemos que existió un nivel de dependencia del promotor teatral, que no necesariamente reproducía el discurso oficial del Estado como lo hemos demostrado.

\section{La vecindad hace teatro: Actores y actrices comunales}

\section{-Jóvenes, estudiantes y trabajadores}

Desde el primer momento en que el proyecto socialdemócrata se plantea el objetivo de la popularización del teatro, advierte que en el sistema de educación secundaria hay un amplio espacio abierto para lograr cumplir este objetivo. En el proyecto que desde un primer momento planteó Fabio Paccioni a las autoridades de culturales del gobierno en el año de 1972 se aprecia este planeamiento ${ }^{20}$. La popularización del teatro cubrió a amplios sectores de la sociedad costarricense de las décadas de1970 y 1980, en parte porque se utilizaron al menos tres políticas culturales esenciales: difusión, mecenazgo y promoción. Sin embargo, creemos que especialmente la promoción teatral estuvo dirigida fundamentalmente hacia una generación particularmente específica: los jóvenes.

Cuando analizamos la lista de informantes que hemos entrevistado para esta investigación, constatamos que para los cuatro grupos estudiados, el promedio de edad de los participantes en los grupos de teatro comunal fue de 20 años. Aunque con el número de informantes entrevistados no podemos establecer una generalización contundente, nos hemos interesado por investigar, a partir de la experiencia de ellos, por las edades de los demás compañeros de los grupos y los resultados de la información constatan nuestra hipótesis: los grupos de teatro comunal del

19 Entrevista realizada a Alexander Valverde, en el Almacén el Gallito, San José, el 17 de octubre del 2007.

20 Cuevas Rafael, El punto sobre la i. Políticas culturales en Costa Rica (1948-1990), (San José, MCJD, 1996) 160 
Valle Central, fueron creados, impulsados y desarrollados por un sector juvenil de la sociedad costarricense. Una de las razones que posibilitaron que los jóvenes estuvieran más predispuestos a involucrarse en estos proyectos obedece a que, en muchos casos, desde el colegio empezaban a leer textos dramáticos que estaban asignados en la materia de español como parte de las políticas de difusión que tenían los programas educativos de este periodo.

Por otro lado, hemos señalado en el capítulo anterior que en varias comunidades del Valle Central existía una tradición escénica muy importante desarrollada por vecinos de mayor edad, cuando algunos promotores intentaron trabajar con estas generaciones mayores, las respuesta de estos vecinos fue de rechazo. Un caso muy interesante sucede con los integrantes del grupo Caña Brava. Ellos empiezan a tener experiencias escénicas con su padre desde los años de 1960, cuando aun eran niños, sin embargo, cuando deciden fundar este grupo a principios de los años de 1980, su padre decide no los acompaña y funda un grupo de teatro que denominaremos de tradición, llamado "Explosión”. Para lo cual se acompaña de vecinos de su generación y algunos jóvenes, esto manifiesta un apego a la tradición escénica de los "cuadros típicos" y la imposibilidad de que esta generación estuviera dispuesta a vincularse a estos proyectos como lo señala Francisco Valverde, integrante de Caña Brava:

Mi papa nunca participó con Caña Brava y nunca nos vio [entre los dos grupos] había una rivalidad $^{21}$

La rivalidad entre los grupos de tradición comunal y los de teatro comunal quedó planteada también en el caso que ya hemos señalado del grupo "Barrio Pobre", donde el problema generacional también se impuso causando la fragmentación del grupo y el surgimiento de "Éxodo".

Otro aspecto importante es que en todos los casos la participación de los jóvenes en los grupos de teatro comunal implicaba un sacrificio importante, pues además de las exigencias de ensayos y presentaciones que exigía la participación en el grupo, los artistas comunales se dedicaban a sus estudios o a sus trabajos particulares, por lo que para seguir esbozando el perfil de los artistas comunales de los grupos de teatro comunal debemos señalar que además de ser jóvenes, también eran trabajadores y estudiantes.

El caso del grupo “Tunante” de San Ramón es muy interesante. Nos ha contado Gerardina Pérez que ella comenzó a participar en el grupo gracias a que laboraba como responsable de salud de su comunidad bajo un programa del Ministerio de Salud que buscaba enlazar a los auxiliares 21 Entrevista realizada a Francisco Valverde, en San Juan de Dios De Desamparados el 17 de noviembre del 2007 
de enfermería con líderes comunales que tenían información acerca de las enfermedades que impactaban a la comunidad. En este proyecto también esa involucrada la Dirección Nacional de Desarrollo de la Comunidad (DINADECO) y es por medio de éste que se les ofrece a estos líderes comunales la oportunidad de participar en unos talleres teatro que posteriormente llevaran a la creación del grupo. Por las características rurales de la comunidad de donde era este grupo, la mayoría de los integrantes trabajaba:

En oficios, algunos [eran] agricultores y otros éramos estudiantes, muy pocos tenían como una profesión, más que todo[eran] oficios. [y los estudiantes eran] de colegio. ${ }^{22}$

En el caso del Taller Alajuelense de Teatro (TAT), el promotor y director del grupo Marco Araya nos señala que este grupo estaba integrado en sumatoria por estudiantes universitarios con la excepción de tres profesionales en las áreas de arquitectura, publicidad y educación primaria..23 En el grupo “Caña Brava” la formación y desarrollo del grupo quedó en manos de una familia, los integrantes que formaron la base del grupo, eran cuatro hermanos que además de dedicarse al grupo teatral trabajaban y estudiaban. De los dos integrantes que entrevistamos, uno es contador y el otro es abogado. Además, es particularmente interesante que, paralelo al trabajo en el grupo Caña Brava, los cuatro se dedicaron a ser payasos profesionales, lo que los hizo que, en un momento determinado, esta actividad más lucrativa desplazase a la actividad del grupo "Caña Brava”. Tres de los cuatro integrantes del grupo formaron la empresa de payasos "Los Pirulos" a la cual todavía se dedican.

Finalmente, el último grupo que analizaremos es “Éxodo”. Este grupo, constituido también por jóvenes es bastante particular. Por un lado, son jóvenes vecinos de los barrios del sur de la capital de San José, pero por otro lado son también trabajadores del MCJD, que se dedican a la promoción teatral o cultural en diversas comunidades.

\section{-“O son mis hijas o el teatro"}

En la entrevista realizada a la promotora cultural y actriz del grupo Éxodo, Lorena Delgado nos señaló lo siguiente:

En ese entonces, a nivel personal yo tenía que tomar una decisión. Yo tenía a mis hijas pequeñas y trabajando en "Diquis Tiquis" era para arriba y para abajo, era [...] sin salario yo no tenía salario en Diquis Tiquis, no ganaba casi nada, nosotros vendíamos a veces funciones pero a veces no y era mínimo lo que se ganaba y yo podía trabajar en "Diquis Tiquis" porque estaba en 22 Entrevista realizada a Gerardina Pérez en San Ramón de Alajuela, el 20 de septiembre del 2007

23 Entrevista realizada a Marco Araya en el CUNA, Alajuela, el 30 de agosto de 2007 
el Ministerio, [...] al yo renunciar al ministerio, ya me quedaba[...] a la derriba a nivel laboral y entonces fue una decisión absolutamente personal y de vida, de que yo tenia una responsabilidad con mis hijas y yo dije: bueno, o son mis hijas o el teatro y en ese momento yo no podía ser una trotamundos del teatro teniendo a tres hijas pequeñas ${ }^{24}$

De manera general, este planteamiento que nos expuso la promotora cultural, formó parte de las realidades que tuvieron que afrontar pronto los grupos de teatro comunal: los jóvenes que habían empezado con estos proyectos en poco tiempo empezaban a contraer responsabilidades como el matrimonio, la paternidad y la maternidad. Esto hizo que la mayoría de los integrantes dejara esta actividad que exigía bastante tiempo. Además, la participación de los artistas vecinales, en los grupos de teatro comunal, en la mayoría de los casos no era lucrativa y esto representó un claro límite a la continuación de los integrantes en el grupo. Esta realidad la enfrentaron tanto los actores vecinales como los promotores teatrales.

Debemos señalar que la dinámica de estos grupos siempre estuvo marcada por la transitoriedad de sus integrantes. En algunos casos los grupos lograban consolidar una base que les permitía mantener continuidad y acumular experiencia, la cual servía como instructivo para los que poco a poco se iban incorporando.

Gerardina Perez fue integrante del grupo "Tunante” solo durante una parte del ciclo de vida de éste. Al consultársele por el motivo de su salida, comentó que obedeció a que se había casado y debía trabajar a tiempo completo en una cooperativa de café lo que le quitaba el tiempo necesario para ensayos y para giras.

En el grupo Caña Brava pasó algo distinto con sus integrantes, ellos empezaron a tener más rentabilidad dedicándose al negocio de los payasos hacia finales de la década de $1980 \mathrm{y}$ principios de 1990. Es importante señalar que este grupo es el único de los que estudiamos que se mantiene vigente hasta el año de 1994, en parte creemos que esto obedeció a que el grupo estaba conformado por hermanos por lo que el vínculo se tornaba más fuerte. Francisco Valverde señaló que a partir de 1990 la mayoría de ellos fueron casándose, sin embargo el matrimonio de él, no significó una limitante para la participación dentro del grupo, incluso en 1994, en un programa de mano de la obra "Un día en la vida de Goyito Valverde", su esposa figura como maquillista del grupo, con lo que ella se terminó incorporando a la dinámica del grupo.

En el caso de Sandra Loría, del grupo TAT, su participación fue más esporádica. Loría recuerda

24 Entrevista realizada a Lorena Delgado en el Barrio la Granja, San Pedro, 25 de septiembre del 2007. 
su experiencia en el TAT más por los dos montajes en los que participó que por otra vinculación con el grupo:

Ellos como que me invitaron, hice el primer montaje, hice el segundo montaje, y ya yo estoy en la universidad y me concentro en mis estudios. Ya después en el 91 entro en artes dramáticas y me dedico a la carrera y de repente en el grupo también hay gente que no es profesional, (profesional en el sentido de las artes escénicas) y ya yo me desvincule de la actividad ${ }^{25}$ En este caso queda patente la característica transitoriedad de algunas personas que participaban en estos grupos, por otro lado, se puede apreciar que Sandra Loría, al dedicarse a los estudios profesionales en la escuela de artes dramáticas, se desinteresa de una actividad en la que la mayoría de los integrantes no son actores profesionales.

En este capítulo hemos venido señalando que las agrupaciones de teatro comunal durante el periodo de estudio, estuvieron constituidas por jóvenes. Este hecho planteaba diversos retos para la permanencia y vigencia de este gran proyecto. Cuando estudiamos las políticas de promoción cultural que desarrolló el MCJD a partir de los promotores culturales, notamos que no se llevó a cabo una evaluación sistemática de la labor promocional que se desarrolló en el transcurso de los años.

Evidentemente un programa que contaba fundamentalmente con jóvenes como su principal material de trabajo debía reconocer que las dinámicas y cambios de este sector de la población son muy volátiles. Esto exigía que, por el lado de la planificación del MCJD, existiera una política de renovación generacional que fuera incorporando nuevos actores a los grupos que se encontraban constituidos, para asegurar su permanencia y esto no sucedió. Si a esto se suma que, por el contrario, la política económica que se implementó en el país impulsó la movilidad laboral de los que en este caso trabajaban como promotores en las comunidades, encontramos todavía más argumentos para apreciar cómo fue que esta actividad fue declinando.

Por otro lado las comunidades poco a poco fueron perdiendo el dinamismo que les había caracterizado durante varias décadas de la segunda mitad del siglo XX. Además, en las comunidades en donde existía un teatro de tradición, éste nunca logró compaginar con las propuestas que los vecinos más jóvenes estaban impulsando a través del teatro comunal. Por el contrario, en los casos donde coexistieron estas dos propuestas escénicas, notamos que entraron en rivalidades y conflictos.

25 Entrevista realizada a Sandra Loría en la CNT, en San Jose, el 3 de noviembre 2007. 


\section{La creación colectiva y la temática política: asistamos a la función de un grupo de teatro comunal.}

\section{-Una nueva práctica teatral}

Hoy hay huelga. Hoy hay huelga. Hoy hay huelga. ¡Huelga!. 1981, Bajaron los precios internacionales del café, 1981, Rodrigo Carazo rompe relaciones con el Fondo Monetario Internacional. ${ }^{26}$

El fragmento anterior es parte de la obra "Huelga" del grupo de teatro Caña Brava. El vecino que estaba acostumbrado a presenciar una función tradicional de teatro, quedaba confundido cuando la obra "Huelga" iniciaba una nueva presentación, los límites entre la realidad y la ficción tendían a disiparse desde el comienzo y el público inmediatamente empezaba a formar parte del acto escénico. Cuatro actores repartidos entre el público, en espacios físicos no convencionales, empezaban a susurrar al oído la frase: ; hoy hay huelga! Inmediatamente otro actor pregonaba el resto del fragmento: Huelga. 1981, Bajaron los precios internacionales del café. 1981, Rodrigo Carazo rompe relaciones con el Fondo Monetario Internacional. Una nueva forma de hacer teatro se consolidaba en los grupos de teatro de comunidad del Valle Central de Costa Rica. ${ }^{27}$ El "nuevo teatro" latinoamericano, surge a partir de la década de 1950, y es un fenómeno que paulatinamente se va ir extendiendo por todo el continente americano. Los nuevos grupos de teatro latinoamericano se proponen incorporar nuevos actores sociales y nuevas realidades en el planteamiento estético de su propuesta artística. ${ }^{28}$

El nuevo teatro latinoamericano encuentra en la creación colectiva de toda la producción del espectáculo su metodología de creación artística. En el proceso de creación colectiva Beatriz Rizk reconoce cinco etapas de creación: la investigación, la elaboración de la obra, la improvisación, el montaje, la presentación. ${ }^{29}$ Estas fases que ha reconocido Rizk no son mecánicas y se pueden invertir, sin embargo es importante reconocer que para el caso de las agrupaciones teatrales comunales del Valle Central de Costa Rica, hemos reconocido la intervención de estas etapas en la creación de las obras.

26 Entrevista realizada a Francisco Valverde, en San Juan de Dios De Desamparados el 17 de noviembre del 2007

27 Entrevista realizada a Francisco Valverde...

28 Risk Beatriz. El Teatro nuevo latinoamericano. Ed Prisma Institute. Minneapolis. 1987.

29 Risk Beatriz. El Teatro nuevo latinoamericano. Ed Prisma Institute. Minneapolis. 198769 a 81 
Como señalamos al principio de esta ponencia, la afluencia de artistas de Suramérica a Costa Rica, durante la década de 1970, dio como resultado que se empezaran a poner en práctica metodologías teatrales que no se conocían en el país. En cuanto al desarrollo de la creación colectiva, es importante destacar el papel del grupo independiente Tierranegra que se fundó en 1973. El director del grupo fue el colombiano Luis Carlos Vásquez, el cual estaba muy influenciado por el trabajo de grupos colombianos con una amplia trayectoria en el campo de la creación colectiva. Como señala Fernando Vinocour, este grupo introdujo nuevas metodologías de trabajo como: la aplicación de recursos corporales y visuales, la producción de un texto y un montaje. Este grupo tenía comisiones de trabajo que articulaban un trabajo intergrupal por medio de fases no comunes en la puesta en escena como: la investigación, la discusión o debates ideológicos, los entrenamientos constantes, la elaboración de los útiles escénicos, entre otras. ${ }^{30}$ En los grupos de teatro comunal analizados apreciamos que los promotores teatrales implementaron la creación colectiva en los grupos de teatro promovidos y al mismo tiempo los vecinos y artistas comunales lograron aprovechar esta oportunidad para construir su propio y autónomo trabajo estético.

\section{-Las obras y los problemas de las comunidades}

Las obras de teatro que pudimos conocer ya sea de forma directa o de forma indirecta, apuntaron generalmente en la dirección de los problemas y las luchas que sus propias comunidades estaban realizando en ese momento. De manera general todos los informantes manifestaron una preocupación social en las temáticas implícitas en las obras de teatro. Es importante señalar que al ser la creación colectiva la metodología que tuvo mayor peso en los grupos estudiados, uno de los problemas al estudiar este fenómeno es que, en la mayoría de los casos no se escribía ningún texto. Hemos podido reconstruir algunas obras de creación colectiva a partir de la memoria y el recuerdo de los creadores de las obras. La creación individual, aunque menos utilizada, también se implementó en algunos grupos, como en el caso del grupo TAT de Alajuela, donde los montajes fueron a partir de autores universales y nacionales. ${ }^{31}$

Ya desde 1981, a solo dos años de que la primera generación del Taller Nacional de Teatro hubiera iniciado sus labores como promotores teatrales en las distintas comunidades del país, se pudo observar a partir de la "Muestra de teatro de comunidades y grupos aficionados", la variedad de obras y temas presentados en la actividad. En el siguiente cuadro reproducimos la información sobre los grupos, las obras y los autores. Véase cuadro 3.

$30 \quad$ Fernando Vinocour editor, La tradición del presente. San José Costa Rica, ed. Perro Azul, 2007p 124

31 Entrevista realizada a Marco Araya... 
De las quince puestas en escena presentadas en dicha muestra, diez son de autor, entre estas, varias adaptaciones de escritores nacionales; cuatro puestas son de creación colectiva; y una de autor anónimo. A pesar de que en esta muestra se percibe una clara tendencia predominante de puestas de autor, podemos señalar algunas hipótesis al respecto: la primera es que las puestas que son adaptaciones teatrales, pueden haber sido trabajadas mediante adaptaciones colectivas y juegos, ya que esta era una metodología que utilizaban los promotores de esta generación para la adaptación de textos no dramáticos. Por otro lado, grupos como Éxodo, que en esta ocasión se presenta con una puesta de autor, que es además integrante del grupo, trabajó constantemente a partir de la creación colectiva. De estas 15 obras presentadas hemos tenido la posibilidad de conocer algunos textos o algunas referencias de las experiencias colectivas de las obras que son de autores colectivos.

También aprovecharemos la información que se desprende del cuadro sobre los grupos, para desarrollar el análisis sobre las obras y las problemáticas de los grupos de teatro comunal que hemos estudiado. Haremos mención sobre algunos trabajos artísticos que se presentaron en esta muestra. Sin embargo, aprovecharemos también para analizar el trabajo desarrollado posteriormente por los grupos. Empecemos primero con los trabajos de autor individual a los que hemos tenido acceso y posteriormente con los trabajos de creación colectiva, donde empezaremos analizando la experiencia rural del grupo Tunante de San Ramón, para posteriormente continuar con las obras de los grupos urbanos: Caña Brava TAT y Éxodo. Finalmente debemos señalar que como tendencia general predomina un estilo neo-costumbrista en la construcción de las obras.

En “Un hombre llamado Juan”, de Yako Serrano, considera la reinterpretación de la identidades nacionales a través de una desmitificación del símbolo de Juan Santamaría, el cual es presentado como un hombre despreocupado, mujeriego y alegre, desmitificando la proyección heroica del símbolo $^{32}$. El tema subyacente en "La parábola de la riqueza” de Víctor Valdelomar es el de la corrupción y de los negocios fraudulentos que lleva a acabo una empresaria, vemos cómo se perfila una crítica a un personaje que representa a la clase empresarial y que manipula a otro personaje de extracción popular, mediante el negocio de traficar pájaros. ${ }^{33}$

En cuanto a la obra del grupo Tunante el promotor señala lo siguiente con respecto a la obra "La Unión de Santa Cachucha”:

La Unión de Santa Cachucha, es una obra que recoge la experiencia de la comunidad de la 32 "Un hombre llamado Juan”. Escena n4,1981

33 "La parábola de la riqueza” Escena n5, 1981 
Unión de San Ramón, la comunidad de San Ramón esta al sector sur de San Ramón [...] nosotros hicimos un montaje que reflejaba la historia de esa comunidad. ¿Cual es la historia de esa comunidad? Esa comunidad nace como un precario[...] como una toma de tierras de campesinos, entonces [...] es una comunidad que fue tomada (sic) por campesinos y montaron sus ranchos y empezaron a organizarse y entonces a partir de ahí surge todo el proceso de montaje de la obra porque uno de los integrantes del teatro Tunante en se momento (era de la comunidad de la Unión)...entonces a raíz de todo el proceso de creación colectiva que desarrollaba el grupo, empezamos a improvisar escenas de la experiencia que había sido la toma de tierras de lo que se llama hoy la Unión en San Ramón entonces con base en esa experiencia se monta la obra "La Unión de Santa Cachucha ${ }^{34}$

Como podemos apreciar en este caso, la realidad de la comunidad ramonense de la Unión quedó plasmada por medio de la creación colectiva, Rubén Garro nos señaló que parte del método usado para la creación de esta obra fue entrevistar a los vecinos que participaron de la toma de tierra. Aunque cuando hablamos con Gerardina Pérez no recordaba con tanta exactitud el tema de la obra, nos señaló que uno de los temas era que "un pueblo tiene que unirse para que la situación mejore" 35 . Particularmente interesante fue cuando la informante se refirió a los métodos que usaron para creación colectiva:

Fue como un poco la creación de todos, la opinión de todos los integrantes y bajo la accesoria del promotor [...] cada quien iba aportando ideas, de lo que le parecía [...] y así nos fuimos poniendo de acuerdo hasta ir montando la obra ${ }^{36}$

Más adelante nos acota que algunas de las estrategias que usaban para este tipo de creaciones colectivas era salir a distintas actividades sociales como romerías para ver los comportamientos de la gente y luego incorporarlo a las obras. Rubén Garro señaló que en determinado momento esta obra fue presenciada por un político de la comunidad que se sintió ofendido, ya que "La Unión de Santa Cachucha”, criticaba las promesas de los políticos tradicionales. ${ }^{37}$

Además de esta obra que se presentó en la "Muestra de Teatro de comunidades y de teatro aficionado" el grupo de teatro Tunante también montó otras obras, entre ellas una de Víctor Valdelomar llamada “Con la luz del Día” que además de presentarse en la misma muestra, se

$34 \quad$ Entrevista realizada a Rubén Garro, en la UCR, 13 de septiembre del 2007

35 Entrevista realizada a Gerardina Pérez en San Ramón de Alajuela, el 20 de septiembre del 2007

36 Ibid

37 Entrevista realizada a Rubén Garro en... 
presentó en la región de San Carlos en actividades de campesinos, ya que el tema de esta obra es también la problemática campesina. Finalmente la otra obra que montó este grupo se llamó “Cuando las marionetas hablaron”, que tocaba el tema de la dominación ideológica.

En cuanto a la experiencia urbana, contamos con la información de las obras del grupo comunal de teatro Caña Brava, de San Juan de Dios de Desamparados, del cual pasaremos a analizar su participación en dicha muestra, junto con otros montajes desarrollados ulteriormente. En "Una cena para el patrón" se cuenta la historia de un empleado que le pide un "vale” o préstamo a su patrón para realizarle una cena al mismo patrón, con el objetivo de obtener un aumento salarial, que al final no logra conseguir. Esta obra esta basada en un texto anónimo, que funcionó como base para construir a partir de la creación colectiva. El integrante Francisco Valverde, nos señaló que esta obra se presentó pocas veces.

Si "Una cena para el patrón” fue una obra que no tuvo mayor suceso, todo lo contrario sucedió con la "Huelga" obra que causó revuelo e impacto en los barrios del sur y también más allá del Valle Central. Alvarenga ha señalado que las huelgas y bloqueos contra las tarifas buses eran muy frecuentes a principios de la década de $1980^{38}$. Sentimos que una de las obras que logró captar el espíritu de esta coyuntura lo constituyó esta obra de creación colectiva. Víctor Valdelomar nos ha mencionado que la estructura estaba basada en tres escenas: la primera era la experiencia cotidiana de viajar en los autobuses, aquí aparecían personajes cotidianos; en la segunda empezaba el conflicto al montarse un personaje de buen vestir que estaba en contra de la huelga, a partir de este enfrentamiento los usuarios decidían reunirse para organizarse y luchar, en este momento el público pasaba a formar parte de la obra, ya que los actores al hacer de líderes comunales se dirigían a los vecinos. Y en la tercera escena se hacía la propuesta del bloqueo de calles, donde, por medio de un juego de pantomimas, se invitaba al público a que cargara piedras para ponerlas en la calle. Otro elemento con que se involucraba al público era por medio de la creación de la "cadena humana", que se utilizaba en la realidad para que los buses no pasaran. ${ }^{39}$ Paralelamente a la obra La huelga, el grupo Caña brava tenia montada otra obra llamada "La abejita haragana", en donde el tema que se toca es el de la solidaridad ya que el personaje principal de esta obra -la abejita haragana- decide no trabajar en la recolección de alimento que se hace en el verano para atravesar el invierno, ante esto, este personaje principal es excluido de la colmena. Sin embargo, después de la lección moralizante que sufre la abejita 38 Alvarenga, Patricia. De vecinos a ciudadanos. Ed. EUNA y EUCR. 2005

39 Entrevista realizada a Víctor Valdelomar en la UCR, San Pedro, 9 de octubre del 2007 
haragana, es aceptada por la colmena. Finalmente la última obra que monta este grupo es "Un día en la vida de Gollito Valverde, donde los temas principales son la movilidad laboral, la inseguridad ciudadana, el alcoholismo y el narcotráfico.

El grupo TAT de Alajuela no tuvo participación en esta muestra porque su constitución se da cuatro años después. Este grupo siguió una estrategia distinta a la de los grupos que hemos estudiado, y se inclinaron por realizar un trabajo a partir de obras de autores internacionales. Al respecto Marco Araya refiere a que se montaron las siguientes obras: "Historias para ser contadas” de Osvaldo Dragún, "La zapatera prodigiosa” de Federico García Lorca y "El vuelo de la grulla” de Ana Istarú”. ${ }^{40}$ En cuanto a su propuesta el director señala lo que era:

Denunciar por denunciar en el sentido de no mezclarse con nada de idiosincrasia politica, lo que más rigió en el grupo fue el arte por el arte, no nos vamos a comprometer con la sociedad para ser los caudillos ni los heroes. Puntualmente nos quedamos con una denuncia.

A través de las obras presentadas por los grupos de teatro comunal hemos podido apreciar la variedad de temas que las agrupaciones trataron. En síntesis, se advierte una preocupación por las problemáticas de las comunidades de donde provenían estos grupos a las que consiguieron plantearles, a través de la creación colectiva en la mayoría de los casos estudiados, una propuesta simbólica y estética de lo que la comunidad y los vecinos estaban reivindicando.

\section{-Las dinámicas internas de los grupos}

En las agrupaciones de teatro comunal que hemos analizado, se dieron procesos y de relaciones internas que influían en la producción y creación final de las obras a partir de los ensayos y de las presentaciones que realizaron, describiremos algunas de las dinámicas que daban en el interior de los grupos:

Antes de que el ensayo diera inicio había que llegar al lugar del ensayo, esto que parece poco relevante, mucho lo era, pues en comunidades rurales como la de San Ramón, el solo hecho de asistir al ensayo se convertía en una gran dificultad, ya que muchos integrantes eran de comunidades bastante alejadas del centro de San Ramón:

Era increíble y lindísimo porque era gente que venía desde (lugares lejanos)[.... Para llegar a la reunión (ensayo) a las 9 de la mañana un domingo, tenían que salir desde las 5 de la mañana [...] y tenían que venir a pie y cruzar en caballo un río, tomar el bus para llegar(al centro) ${ }^{41}$ Después de llegar al lugar de ensayo, que en el caso de Tunante era en unas aulas que prestaba 40 Entrevista a Marco Araya realizada en ...

41 Entrevista realizada a Rubén Garro... 
la asociación regional de salud comunitaria, se empezaba con calentamientos, ejercicios de formación actoral, de entrenamiento físico, y si se estaba en el proceso de montaje se hacían ensayos generales. El grupo también realizaba investigaciones de observación participante donde los integrantes salían a ciertos espacios públicos para observar cómo se comportaban los vecinos en ciertas actividades particulares, posteriormente se traía esta información al ensayo y se realizaban improvisaciones a partir de estos materiales. Estas actividades de investigación son recordadas por la Gerardina Pérez como giras de reconocimiento para conocer la cultura y costumbres del pueblo y además agrega que en las giras se realizaban almuerzos grupales.

En el caso de los teatros comunales urbanos, llegar al ensayo no representaba tantas dificultades como lo era en el caso rural. En cuanto a la dinámica de los ensayos, esta no varió significativamente de la que realizaba el grupo Tunante. El TAT de Alajuela ensayaba los domingos en la mañana en el museo Histórico Juan Santamaría y además de las técnicas que ya hemos mencionado también hacían calentamientos vocales. El grupo Caña Brava por su parte, realizaba sus ensayos en una bodega de pinturas ubicada en la Uruca, de uno de los integrantes y, al igual que los demás grupos, lo hacia los sábados o los domingos.

En cuanto a la escenografía, utilería y vestuarios utilizaos por los grupos de teatro comunal analizados, todos sin excepción nos han señalado que siempre se buscó que fueran los mínimos y de bajo costo, esto se debe a que el financiamiento de los grupos corría por parte de los mismos integrantes, además cuando había alguna gira a otra comunidad, el tener pocos elementos escenográficos les permitía mayor comodidad de transporte. En el caso del Caña Brava, los integrantes nos comentaban que cuando presentaban la "Huelga" lo único que utilizan era un tubo plástico que representaba la barra que tienen los buses para que la gente que va de pie se sostenga. En síntesis debemos señalar los elementos escenografitos utilizados por los grupos de teatro comunal eran escasos y además de bajo costo. 


\section{Nuevos espacios-teatrales y nuevos públicos vecinales. De los teatros a los salones comunales.}

\section{-¿EI fin de la sala de teatro como único espacio de representación?}

El desarrollo del nuevo teatro latinoamericano, surge con la intención de generar un acercamiento con públicos que anteriormente no eran tomados en cuenta por los formatos tradicionales de la representación escénica teatral. Todo lo anterior hace que la sala de teatro tradicional empiece a perder sentido para el nuevo teatro latinoamericano y de esta manera comienza a imponerse la necesidad de trabajar a partir de otros espacios escénicos más inmediatos y más cercanos a la nueva realidad teatral. Como consecuencia se da un tránsito de espacios cerrados a espacios abiertos o bien de espacios cerrados a otro tipo de espacios cerrados, que permiten una mayor familiaridad con el nuevo público. Por otro lado, las propuestas que surgen a partir de la creación colectiva buscan que el mismo público sea parte fundamental de la obra y que incluso la modifique, en última instancia la sala tradicional de teatro se convertía en una limitante para que esto sucediera, ya que las la estructura de la sala no permite una relación cercana con el público.

En el caso de la experiencia histórica del fenómeno teatral costarricense durante el periodo en análisis, debemos señalar que las salas de teatro nunca perdieron totalmente su función como espacio tradicional de la representación teatral. Sin embargo, con toda la influencia de las nuevas propuestas teatrales, se dio una marcada tendencia a retar la funcionalidad de las salas tradicionales, sobre todo cuando la intención era atraer o bien identificarse con públicos no tradicionales. Uno de los grupos independientes que abrió camino en esta dirección fue el "Teatro Carpa”, que, como lo indica su nombre, utilizaba una gran carpa de circo para andar presentándose en diferentes comunidades.

Aunque los grupos de teatro comunal en algunas ocasiones utilizaron salas teatro tradicional, sobre todo cuando eran invitados a festivales de teatro comunal promovidos por el Estado o por alguna Municipalidad, su espacio natural de representación lo constituyen lugares atípicos que surgen ante la necesidad de realizar las metas que estos grupos comunales se proponían. En este sentido es importante señalar que dado el origen de los montajes a partir de las problemáticas comunales, el público al cual pretenden acercarse son los mismos vecinos de la comunidad a la que pertenecen. Como es evidente, la infraestructura teatral, salvo contadas excepciones, se concentró alrededor del centro de la capital de San José, y las comunidades no contaban con teatros. 
Por esta razón, el ámbito de representación característico de las agrupaciones de teatro comunal fue el salón comunal, que para esta coyuntura existían en muchas comunidades. Este espacio fue tan importante que en algunos casos los salones estaban adaptados con alguna infraestructura básica para la representación escénica como recuerda Víctor Valdelomar:

Inclusive yo recuerdo un salón comunal de San Rafael abajo de Desamparados, que lo tenía un grupo de jóvenes, y ellos habían adaptado el salón con ciertas luces y cosas, telones y cuestiones para mantener una actividad teatral constante ahí. ${ }^{42}$

En el caso del grupo Tunante de San Ramón, tanto el promotor como la ex integrante del grupo, se refieren al salón comunal como el espacio tradicional de representación del grupo. En el caso del grupo TAT de Alajuela, el lugar donde se presentaba con mayor frecuencia era en el auditorio del Museo Juan Santamaría, sin embargo Sandra Loría recuerda que uno de los montajes en los que participó fue en las instalaciones de la antigua estación de trenes. El objetivo de presentarse en este lugar era intervenir en la cotidianeidad de la comunidad y, al mismo tiempo, aprovechar el espacio al aire libre para invitar a la comunidad a presenciar el espectáculo. En la experiencia que recuerdan los integrantes del grupo Caña Brava, está presente el salón de actos de la escuela de la comunidad de San Juan de Dios de Desamparados, como uno de los lugares donde más funciones dieron, esto se puede explicar porque desde su experiencia en la representación de las estampas típicas, en las veladas escolares, este lugar se convirtió en un espacio imprescindible para los grupos de teatro de tradición.

Además de los salones comunales, otros espacios que fueron aprovechados por los grupos de teatro comunal fueron los atrios de las iglesias, los "turnos" o festejos patronales, las calles públicas, las instalaciones deportivas, los parques públicos, las instalaciones de sindicatos o de partidos políticos, las bibliotecas e instalaciones públicas como casas de la cultura o museos. En fin, los grupos de teatro comunal aprovecharon cualquier espacio, que asegurara la participación del público para el que habían sido creadas las obras y no tuvieron ningún reparo en presentarse, tanto en teatros como en salones comunales o en alguno de los lugares mencionados.

\section{-El público comunal.}

Así como se plantea el surgimiento de un "nuevo teatro latinoamericano", se plantea también el surgimiento de un "nuevo público latinoamericano", integrado por sectores sub alternos. El Estado, a través del Instituto Nacional de Vivienda y Urbanismo (INVU), desarrolla varios

42 Entrevista realizada a Víctor Valdelomar en la UCR, San Pedro, 9 de octubre del 2007 
proyectos habitacionales para estos sectores sociales. De esta forma la ciudad se ira expandiendo hacia los barios del sur con el propósito de albergar a esta nueva población. Algunos de los grupos de teatro comunal que hemos analizado para el periodo, provienen de estas barriadas del sur de la capital, como en el caso de Éxodo y Caña Brava. En el caso de la experiencia de San Ramón, los vecinos que se van a constituir en publico serán en mayoría campesinos.

Si se había creado un público nuevo que empezaba a asistir a las presentaciones de los grupos de teatro comunal, queremos saber que estrategias desarrollaron las agrupaciones de teatro comunal para a traer al nuevo público a las presentaciones que realizaban constantemente. En el caso del grupo Tunante de San Ramón, las estrategias desarrolladas por el grupo fueron la introducción de volantes donde se anunciaba sobre los espectáculos específicos. Este grupo también aprovecho el sistema de comunidades que integraban los programas del Proyecto de promoción de la salud pública que desarrollaba el Ministerio de Salud en esta región. Por eso hemos apreciado como el grupo se movía por las comunidades del cantón aprovechando la red de comunidades. Señala Rubén Garro que la reacción del público ante las presentaciones fue muy efusiva y además estuvo marcada por la diversión que permite la reflexión, pues las obras generaron identificaciones con la realidad que cotidianamente vivía el público.

En el caso de los grupos urbanos, el proceso de contacto para atraer al público utilizo varias estrategias como la repartición de volantes en la comunidad, la pega de afiches en lugares públicos. En los casos en que la relación con el cura de la comunidad fue buena, el final de las misas representa un importante espacio que los grupos de teatro no desaprovecharon, ya sea anunciando las presentaciones con el cura o aprovechando la salida de misa para repartir volantes. En general como sucedió, también en el caso del grupo de San Ramón, los grupos de teatro comunal urbanos a aprovecharon la infraestructura y las redes comunitarias de organizaciones comunales. En el caso del grupo TAT, de Alajuela el director nos señalo que las funciones siempre tuvieron una buena asistencia, por su parte la integrante Sandra Loría, ${ }^{43}$ nos comentaba que las familias de los integrantes eran también las encargadas de llevar publico a las presentaciones. Entre las estrategias que ensayo este grupo, estuvo también la de utilizar espacios no tradicionales como la antigua estación de trenes, aquí la cercanía con la comunidad permitía atraer nuevos públicos a través de la intervención en la cotidianeidad

43 Entrevista realizada a Sandra Loría en la CNT, en San Jose, el 3 de noviembre 2007. 


\section{-Nuevas dinámicas del fenómeno teatral: el teatro foro.}

En los grupos que trabajaron a partir de la creación colectiva, el público se constituye en un elemento muy importante del fenómeno teatral, incluso cada nuevo espectáculo es un gran reto porque los grupos siempre están anuentes a que las obras estén en proceso de constante modificación, por parte del público. En este tipo de representación escénica, una misma obra puede variar mucho dependiendo de cada público. En este sentido, la participación de actores y espectadores en un foro de discusión fue un recurso utilizado bajo esa misma idea de convertir al espectador en un elemento determinante de la creación artística.

Debemos señalar que para el caso de los grupos de teatro comunal que analizamos, no siempre esta estrategia fue utilizada por todos los grupos. Sin embargo, en algunos casos de agrupaciones teatrales que no realizaron esta dinámica, se ensayaron otras que permitieron que el público opinara acerca de lo que le parecía la obra que estaba viendo e incluso hasta otorgándole la posibilidad de modificarla. Como el caso que plantearemos más delante de la obra La huelga del grupo Caña brava.

En el caso del grupo Éxodo de los barrios del Sur, Lorena Delgado nos ha señalado que durante su participación con este grupo de teatro, si se implemento el foro o la discusión al final de la representación:

Nosotros hacíamos lo que se llama un foro al final y le preguntábamos a la gente que ¿Qué pensaba de la obra? ¿Cómo la veía? ¿Que había entendido que no había entendido? Entonces era como muy dinámico porque podíamos cambiarlo, verdad. Cambiar algo que sentíamos que la gente no quedaba claro. ${ }^{44}$

En el caso de esta agrupación teatral, que como ya anteriormente hemos señalado funciono como laboratorio para sus integrantes y como influencia para los demás proyectos que desarrollaron sus integrantes por separado, si se utilizo este elemento de discusión con la comunidad.

En el caso del grupo Tunante de San Ramón de Alajuela también se implemento esta dinámica. Al consultársele a Geradina Pérez por la existencia de este elemento respondió lo siguiente Si al final con la misma gente que estaba ahi viendo la obra se les preguntaba que le habia parecido, que si en realidad usted siente que es parte de usted mismo, la vida que usted ha llevado, las costumbres del pueblo y todo eso, y coincidía porque la gente estaba de acuerdo que sí. ${ }^{45}$

44 Entrevista realizada a Lorena Delgado en el Barrio la Granja, San Pedro, 25 de septiembre del 2007

45 Entrevista realizada a Gerardina Pérez en San Ramón de Alajuela, el 20 de septiembre del 2007 
Además de que efectivamente si utilizaron este elemento, observamos que desde el recuerdo de Doña Gerardina Pérez, los espectadores si se sentían identificados por una obra en la cual sabemos que se hacían críticas muy fuertes a la vida del trabajador campesino.

Por otro lado encontramos grupos donde nos señalaron que no se implemento este elemento. Sin embargo, aquí hay que hacer en una distinción entre el grupo TAT de Alajuela y el grupo Caña brava de San Juan de Dios de Desamparados. En el primero de los grupos, por la dinámica de su trabajo que se realizo a partir de obras de autores universales o nacionales, estos no podían modificar las obras con la opinión o participación del público.

El caso de Caña brava es singularmente interesante porque a pesar de lo que nos señalo uno de sus fundadores, Francisco Valverde, de que no realizaban esta actividad, esto no impedía que en obras como La huelga, esta tuviera estructurada para que el público participara y opinara en el transcurso de la obra. Es un fenómeno curioso donde el espectador que había participado de la huelga real, perdía el horizonte entre realidad y ficción en medio de la obra, lo que permitía que los actores y el grupo en general estuvieran atentos a recordar las acotaciones que hacían los espectadores en medio del espectáculo, pudiendo reconstruir ulteriormente la obra. Esto coincide con el señalamiento que nos han hecho los integrantes del grupo de que al principio esta obra duraba solo nueve minutos y que después de vario tiempo transcurrido entre representaciones, la obra termino durando una hora.

Entonces la comunidad se veía en el trabajo de nosotros, se veía como que era la vivencia de ellos, al extremo de que se sentían que faltaba más, porque en la obra llegábamos nosotros y entrevistábamos al publico como si fuera parte de esa huelga: "Señores ¿como se sienten, como se sienten en estos momentos que ha llegado las autoridades?”, entonces los metíamos, el público era un personaje más en la [obra]... cuando terminaba la obra quedaba como una sensación como de que verdad había existido...como que había pasado una huelga. Como que la gente se sentía metida, inmersa: como que, sí es cierto, hubo huelga aquí. Entonces había una reacción de todo tipo de: "si es una injusticia eso no puede darse", la gente decía: "eso no puede darse, hay que acabar, no puede darse esa represión, no puede”, así era la expresión de la gente. ${ }^{46}$

46 Entrevista realizada a Francisco Valverde, en San Juan de Dios De Desamparados el 17 de noviembre del 2007 
Como vemos esta estrategia permitió que a pesar de que no hubiera un foro al final de la obra, el publico opinara acerca de lo visto en comparación con lo que su experiencia personal había vivido en la huelgas y cierres de calles por las mejoras de los servicios de autobuses.

\section{Conclusión General}

Los grupos de teatro comunal, en algunas comunidades del valle central, fueron un referente simbólico para las mismas comunidades, que en muchos casos se encontraban enfrascadas en diferentes luchas por el mejoramiento de servicios públicos y privados, contra el aumento de estos mismos servicios y por en desarrollo de una ciudadanía activa y participativa preocupada por las problemáticas locales, regionales y nacionales.

Las agrupaciones de teatro vecinal, a partir del recurso artístico, lograron representar el sentimiento general de las experiencias de los vecinos, ya sea de sus propias comunidades o de otras donde asistían por invitación. En el planteamiento temático de algunas de las obras se advierte que estos también estaban moviéndose en estos tres espacios, el local, o sea el barrio de donde provienen, el regional, como sucedió con la experiencia que desarrollo la obra La Huelga en los barrios del sur, o nacionales, donde se trataron temáticas como la movilidad labora, la inseguridad ciudadana, entre otras.

Las agrupaciones de teatro vecinal fueron un evento al interno de las comunidades, ya que los grupos de teatro comunal se presentaban a partir de espacios no convencionales que rompían con las tradicionales visiones del fenómeno teatral. Nos hemos dado cuenta que en algunas comunidades de las que estudiamos, existió una práctica escénica previa a los grupos. Esto nos conduce a sugerir que la experiencia estética-vecinal en relación con el fenómeno escénico, no fue un acontecimiento que introdujo la política cultural del Ministerio de Cultura Juventud y Deportes. En buena medida, los grupos teatro de tradición, fueron también los que posibilitaron el surgimiento, de los grupos de teatro vecinal, sin embargo al surgir los segundos, encontramos una rivalidad entre ambos estilos de representación escénica.

Al principio, hemos estudiado el surgimiento, así como la desaparición de este tipo de agrupaciones artísticas en el Valle Central de Costa Rica. Son dos vías por las que surgieron los grupos de teatro comunal. En la primera intervienen: las nuevas políticas culturales del Estado costarricense, una fuerte organización a nivel comunal con presencia de distintos grupos de 
izquierda, la contribución de los artistas exiliados suramericanos Y en la segunda, juega un papel determinante la experiencia heredada de los grupos de tradición.

Son una serie de factores bastante heterogéneos que se confabulan para que los grupos de teatro comunal dejen de funcionar a final de la década de 1980 y principios de 1990. entre ellas cabe mencionar el decaimiento y crisis de las agrupaciones comunales para 1990, el cambio de política económica que implementaba el Estado, fue de paticular importancia las movilidades laborales, la caída de motivaciones externas como el derrumbamiento de la URRS, entre otras. También hemos estudiado a los artistas comunales que participaron en los grupos de teatro comunal. Nos hemos dado cuenta que dicho proyecto fue realizado por un sector juvenil de la sociedad costarricense, tanto los promotores del MCJD como los mismos artistas comunales que paralelamente al trabajo que desarrollaban al interno de los grupo de teatro, llevaban acabo estudios, secundario o universitarios así como trabajos varios. Una de las realidades que tuvieron que confrontar los grupos de teatro comunal es que muy tempranamente los artistas tenían que dejar los grupos porque adquirían compromisos matrimoniales, paternales o maternales al mismo tiempo el MCJD no desarrollo una política cultural que fuera capaza de integra nuevos integrantes a los grupos de teatro vecinal.

Por otro lado, hemos estudiado propiamente a los grupos de teatro comunal desde algunos de los elementos que los constituían. Nos hemos dado cuanta que la metodología de la creación colectiva fue un componente esencial en el desarrollo creativo de los grupos aunque no necesariamente el único, pues también coexistió la creación individual y el montaje de obras de autores universales o nacionales. Analizamos también el tipo de obras y temáticas que desarrollaron estas agrupaciones y encontramos que en todas sin excepción existió la preocupación de plantear temáticas sociales que se plantearon de diferentes formas. Por último, nos metimos al interno de los grupos para apreciar cuales eran algunas de las dinámicas que desarrollaron internamente.

Finalmente, analizamos las modificaciones que plantearon a la práctica teatral las agrupaciones de teatro comunal, en este sentido ubicamos nuevos espacios de representación escénica no tradicionales. Estudiamos también al nuevo publico que integro este fenómeno teatral comunitario: los vecinos de sus propias comunidades los cuales fueron integrados a los espectáculos pues en escena se simbolizaban las experiencias comunales de estos vecinos. Por ultimo analizamos la introducción dentro del espectáculo de elementos como el foro de discusión de la obra y la temática al final del espectáculo. El uso de este elemento no fue de uso generalizado, sin embargo se emplearon estrategias distintas durante el transcurso del espectáculo que le permitían 
al publico participar activamente en el desarrollo de la acción dramática.

Las agrupaciones de teatro vecinal marcaron un precedente en la historia cultural y social del país. Del mismo modo, estuvieron inscritos dentro de la tradición histórica de un nuevo teatro latinoamericano que surgió ante la necesidad de integrar las experiencias que viven los actores de la vida real de las sociedades latinoamericanas como de otras regiones. Estas agrupaciones artísticas supieron estar atentas al pulso que marcaba la época para provocar un tipo de teatro que vinculo a amplios sectores y clases sociales que anteriormente no habían tenido la oportunidad del goce estético. Las agrupaciones de teatro vecinal son otra faceta más de la amplia efervescencia que gozaron las comunidades costarricense durante una gran parte de la segunda mitad del siglo $\mathrm{XX}$.

\section{Cuadro 1}

Lugares de promoción según promotores de la CNT y estudiantes Del TNT. 1980

\begin{tabular}{ll}
\hline Promotores de la CNT & Alumnos de la TNT \\
\hline Aldea Santa Ana & Tierra Blanca de Cartago \\
Aldea Rositer Carballo & Niños Cárcel de Menores \\
Taller de artes plasticas MCJD & Taller para maestros escol y pre - escol \\
Patronato Nacional de la Infancia & Grupo com de jóvenes Cedros San_Pedro \\
Gravillas & Grupo comu jóvenes Heredia centro \\
Cartago centro y el Guarco & Grupo com niños casa cult Paso Ancho \\
Instituto Nacional de Seguros & Grup. comunal niños y jóv Desamparados \\
Alajuela casa de la cultura & \\
Instituto Costarricense de electricidad & \\
Liceo de Señoritas y liceo de Costa Rica & \\
Salón comunal de San Ramón & \\
Tilarán & \\
Limón & \\
Nicoya & \\
Puntarenas & \\
San Isidro del General & \\
Heredia & \\
San Carlos & \\
Barrio Cristo Rey & \\
Barrio González Víquez & \\
\hline
\end{tabular}

Fuente: Compañía Nacional de Teatro. Programa de Trabajo Repertorio 1980. Escena n3, p3 


\section{Cuadro 2}

Lugar de procedencia de los grupos de teatro comunal y aficionado que se presentaron en la muestra de teatro de comunidad y grupos aficionados. 1981

\begin{tabular}{lllll}
\hline GRUPO: & LUGAR: & GRUPO: & LUGAR: \\
\hline Tunante & San Ramón & Cristo rey & San José \\
Temuco & Cartago & Escazú & Escazú & \\
Santamaría & Alajuela & Reyco & San José & \\
Fanal & Alajuela & Caña Brava & Desamparados & \\
Teatro-TEC & Cartago & Éxodo & San José & San Isidro del \\
INS (niños) & San José & Carmen Lyra & General & \\
ESC. Goicoechea & Paraíso & & & \\
\hline
\end{tabular}

Fuente: Hacia un Teatro Popular Costarricense, Escena n6, p20 


\section{Cuadro 3}

Grupos, obras y autores presentados en la Muestra de teatro de comunidades y grupos aficionados, junio de 1981

\begin{tabular}{lll}
\hline Grupo & Obra & Autor \\
\hline Tunante & $\begin{array}{l}\text { 1-La unión de Santa } \\
\text { cachucha }\end{array}$ & Creación colectiva \\
& $\begin{array}{l}\text { 2-Con la luz del día } \\
\text { Temuca }\end{array}$ & V. Valdelomar \\
Santamaría & La farsa de Pethelin & F. Dobles \\
Fanal & Un hombrenimo llamado Juan & Yako serrano \\
& El fantoche Lusitano & Meter Waiss \\
Teatro-Tec & Ensueño de Navidad & Carmen Lyra \\
INS ( niños) & Juegos de niños & Jaime Silva \\
Esc. Goicoechea & 1 Tío conejo y tío coyote & Carmen Lyra \\
Cristo Rey & 2 Pantomimas & Creación colectiva \\
& & \\
Escazú & & Creación colectiva \\
Reyco & La consentida & Manuel Galich \\
Caña Brava & Ropa de Teatro & Creación colectiva \\
& Una cena para el patrón & \\
Éxodo & La parábola de la riqueza & V. Valdelomar \\
Carmen Lyra & El chispero & A. Sieveking \\
\hline
\end{tabular}

Fuente: la misma del Cuadro 2 